\title{
A Review on Delay Prediction Techniques in MANET
}

\author{
Harshita Tuli \\ Research Scholar (CS) \\ Jaipur National University \\ Jaipur
}

\author{
Sanjay Kumar, Ph.D. \\ Associate Professor \\ Jaipur National University \\ Jaipur
}

\begin{abstract}
For wireless ad hoc networks, routing is much more complex than in traditional wireless systems, due to the lack of centralized control, infrastructure less nature and knowledge of a predetermined topology. The infrastructure less nature of MANETs makes it difficult to ensure the reliability of packet delivery over multi-hop routes. So, end to end delay in packet delivery is an important QoS metric in ad-hoc network routing. End-to-End delay comprised of delay involved in transmission, propagation and processing. Estimation of end to end packet delay in mobile ad-hoc network is complex because it depends on many influential factors as path length from source to destination, average neighbors of intermediate hops, link expiration time, mobility, bandwidth and interference etc. This paper focusses on different methods adopted by different scientists for estimation and prediction of delay.
\end{abstract}

\section{General Terms}

MANETS, Artificial Neural Network, Bandwidth allocation

\section{Keywords}

MANETS, Artificial Neural Network, End to end delay, Quality of Service (QoS)

\section{INTRODUCTION}

MOBILE Ad hoc networks (MANETs) are composed of mobile nodes connected by wireless links. All nodes can freely and dynamically self-organize into arbitrary and temporary "Ad Hoc" network topologies to serve real time applications. MANETs have been an area for active research over the past few years due to their potentially widespread application in military and civilian communications. They do not use any infrastructure such as base station or router. The lack of infrastructure makes it difficult to ensure the reliability of packet delivery over multi-hop routes also because of its infrastructure less nature every node performs as a host as well as a router. Moreover a node is in charge of routing information between its neighbors, contributing and maintaining connectivity of the network. In (MANETs), a source node must rely on other nodes to forward its packets on Multi-hop routes to the destination. For wireless ad hoc networks, routing is much more complex than in traditional wireless systems, due to the lack of centralized control and knowledge of a predetermined topology. Thus, in a MANET, quality of service is of prime concern. In order to provide quality delivery to real time applications, it is imperative that ad-hoc networks provide quality of service (QoS) in terms of throughput, delay, jitter, reliability etc. End to End delay is an important QoS metric in ad-hoc network routing. End-to-End delay refers to summation of delay involved in transmission, propagation and processing of a data packet. Transmission delay occurs due to link breakage between the nodes reasons for which are mobility, limited battery of the nodes, topological ambiguity or chosen routing protocol. Queuing delay is a function of queue length and determined by traffic load and available bandwidth. Processing delay is dependent on receiver's capacity to process each packet in its queue.

Other than Delay, loss of packets is also a big issue in case of adhoc networks. A sent packet may not reach the destination because of mobility of node or misbehavior. Many nodes misbehave in forwarding the data packet to their neighbors in order to save their power results in packet dropping. But as MANETs are used in the areas like defense assurance of timely delivery of packet is important. Once, delay is predicted the MANETs can be made delay tolerant which in turn makes the network more secure and efficient.

Estimation of end to end packet delay in mobile ad-hoc network is also complex because transmission, queuing and processing delays have to be considered, which in turn depends on many influential factors as path length from source to destination, average neighbors of intermediate hops, link expiration time, mobility and interference etc. This paper is a review study of different methods adopted by different scientists for estimation of delay and experiments done so far to make the network delay tolerant.

\section{BACKGROUND}

Many researchers have tried to put light on different factors affecting packet delivery in MANETS. Mobility has observed to be the most important and influential factor affecting the timely delivery of a data packet. Because of nodes mobility, some of links on the shortest path may fail as soon as the path is established. So, in papers [9],[10],[11] authors have focused on mobility prediction of nodes through different ways in order to ensure the packet delivery. In [9] authors have proposed a mobility prediction method based on a recurrent neural network. In [10] Mansour Sheikhan and Ehsan Hemmati, have done a study on a transient chaotic neural network (TCNN) and presented multipath routing algorithm in MANETs. They proposed that each node in the network can be equipped with a neural network, and all the network nodes can be trained and used to obtain optimal or suboptimal high reliable disjoint paths. In [11], by Hadi Noureddine, et al they have proposed a link lifetime prediction method for greedy and contention-based routing. Mobility directly affects link quality between the nodes which in turn delays the packet delivery or may never deliver it. So [19] and [20] authors have focused on link quality prediction and estimation.

Delay throughput tradeoffs in mobile ad hoc networks comprising heterogeneous nodes with restricted mobility [7] and [12]. In particular, the situation in which each node moves around its own home-point according to a restricted mobility is considered.

In paper [4] authors have tried to deal with packet dropping problem of nodes (selfishness of nodes) with a new method in which given a path, a context-free protocol can transmit packets through it, without knowing whether the intermediate nodes are selfish or not. In this method, the data of a packet is 
encrypted and the identity of the destination is revealed after all nodes forwarded the packet cooperatively. Detection of packet forwarding misbehavior due to selfish nodes has also been proposed in [18].

If a packet is lost due to link breakage, then the cost of sending a packet can be more critical than hop counts in determining the end-to end delay. So in [6] authors have presented an analytical model for average end-to-end delay that takes into account the packet arrival process, back off and collision avoidance mechanisms of random access MAC between a pair of source and destination and compares the end-to-end delay experienced by a AODV protocol. In paper [21] packet dropping problem due to link breakage and low battery issues has also been taken into account.

Zhihao Guo et al have focused on a delay prediction mechanism and its integration with a MANET proactive routing protocol. They have demonstrated their approach of predicting mean queuing delay as a non-stationary time series using appropriate neural network models [8]. In [2] Jyoti Prakash Singh et al have tried to evaluate the applicability and capability of artificial neural network for prediction of end-toend packet delay in mobile ad hoc network environment. They have used path length and average number of neighbors between source destination pair as input parameters to calculate the delay. In their study, they have developed two models based on Radial Basis Function (RBF) network and Generalized Regression Neural Network (GRNN). Further in [13] J P Singh et al has tried to evaluate the applicability and capability of fuzzy logic based model for prediction of end-toend packet delay in mobile ad hoc network environment. In $[16],[17]$ authors have used neural network based method for packet routing.

Other than predicting the delay the network must be made delay tolerant in order to deliver the packet in time. The Delay-Tolerant Network Research Group (DTNRG) in paper [3] has proposed architecture to support messaging in delaytolerant applications. The architecture presented in this paper consists of an overlay, called the bundle layer. A bundle is defined as a number of messages to be delivered together. The bundle layer stores and forwards bundles between DTN nodes, which facilitates hop-by-hop reliability and retransmission. Existing routing algorithms for Delay Tolerant Networks (DTNs) assume that nodes are willing to forward packets for others. But In the real world, they are willing to forward packets for nodes with which they have social ties but not others, and such willingness varies with the strength of the social tie. Thus in [5] Social Selfishness Aware Routing (SSAR) algorithm to allow user selfishness and provide better routing performance has been proposed. To select a forwarding node, SSAR considers both users' willingness to forward and their contact opportunity, resulting in a better forwarding strategy than purely contact-based approaches using Multiple Knapsack Problem with Assignment Restrictions.

\section{FINDINGS}

After reviewing the available research it has been observed that accurate prediction of delay is very important and the techniques used so far have done well in this field. For a given network if a packet is to be transferred from one node to another the shortest path with minimum intermediate hops should be discovered that the packet should travel on. Also due to heavy traffic load from a particular node the shortest path may not provide surety of packet delivery. Moreover node misbehavior may exist. Nodes may delay or may not deliver the packet sent to them in order to save their battery life. Nodes may participate in the route discovery and maintenance processes but refuse to forward data packets. All these problems are categorized under:

\subsection{Transmission Delay}

Transmission delay is the direct function of mobility of nodes and path length. Mobility of nodes can be random or can be simulated under some mobility model and path length can be determined by intermediate hops from source to destination on the network. Because of nodes mobility, some of links on the shortest path may fail as soon as the path is established. So, routing based on selecting the shortest path may not prove to be efficient in ensuring packet delivery. Also congestion level over the network will delay the transmission of the packet. Mobility can be predicted by predicting link quality or estimating the link quality. Authors have proposed a mobility prediction method based on different neural networks independent of the mobility model used by nodes, giving different results. Many research activities have been conducted to determine Link Expiration Time and Path Expiration Time.

\subsubsection{Link Expiration Time:}

Link lifetime represents the time nodes remain within the transmission range of each other.

\subsubsection{Path Expiration Time:}

Path expiration time is the duration for which a particular set of nodes, forming a path, remain within the transmission range of each other. If even a single node leaves the network the path expires.

\subsection{Queuing Delay}

Queuing delay is directly dependent on congestion level. Reduced average queuing delay indicates reduced overall congestion level, in other words, more balanced traffic among the nodes. Also, as each node has a different traffic load, therefore the average number of packets in the queue and the associated queuing delay at each node is different. Secondly, as the number of neighbor nodes as well as their traffic patterns are different, and thus, nodes that have more number of active neighbors may encounter more collisions. Collision may lead to packet loss. If some of the heavily loaded nodes fall on the shortest route, it may actually introduce longer endto-end delay, even though the number of hops along the chosen route is minimum furthermore, if some of the heavily loaded nodes are congested, it may lead to massive packet drop rates and subsequent retransmission. Also queuing delay depends on available bandwidth.

\subsection{Delay Prediction}

Prediction of delay can be done through many ways. In the above literature the prediction is done through ANN approach and fuzzy logics. [13] Jyoti Prakash Singh et al have tried to evaluate the applicability and capability of artificial neural network for prediction of end-to-end packet delay in mobile ad hoc network environment. J P singh, prabhat kumar and sunil kumar singh have tried to evaluate the applicability and capability of fuzzy logic based model for prediction of end-toend packet delay in mobile ad hoc network environment. [13] Through experiments, they have found that trapezoidal fuzzy number based model gives a reasonably good prediction result in terms of various performance evaluation criterion. [16], [17] authors have used neural network based method for packet routing. Prediction of delay can be done by many ways out of which ANN approach has given better results than any other. 
It has been observed that queuing delay and transmission delay are inter- related. As path length will affect transmission of packets also congestion level on the shortest path (queuing delay) cannot be over looked. Moreover a node may be in the range of two different wireless networks and the traffic through that node may suffer bottleneck situation. Also nodes on the network have to share the bandwidth available for a particular flow of information. If two nodes are in range of a wireless network but are communicating with other two different nodes on different networks then have to share the bandwidth available to them. In that communication one node may have to wait if the other has already started the flow. Then, queuing delay on the first node will be higher affecting the overall transmission than the second one. For this bandwidth allocation algorithms need to be studied. The disconnected nature and the lack of end-to-end connectivity between nodes mean that the communication must be delaytolerant by creating a buffer of packets or the bundle layer. Delay tolerant networks involve the concept of bundle layer for ensuring timely delivery of packets and avoiding the overhead of retransmission.

\section{CONCLUSION}

In this paper different approaches discussed by different researchers to study MANETs and delay prediction are reviewed. So far it has been observed that delay is a dynamic metric. Out of transmission, queuing and processing delay queuing delay involves much overhead due to the scarcely available and sharing of bandwidth. Delay can be predicted using different ANN models due to its self-learning capability. There are several features of neural networks that make them valuable and attractive for forecasting. Some of the advantages of modelling using artificial neural networks are:

(i) Artificial neural networks provide the potential to identify and classify network activity based on limited and incomplete data sources. Neural networks are able to learn from examples and respond to subtle functional relationships within the data.

(ii) The most important feature of neural networks is that they can generalize. After learning the data presented to them, neural networks can often predict an event.

(iii) Neural networks approximates with high accuracy.

(iv) Neural networks can be used when only limited data sets are available.

Because of all these characteristics of artificial neural network, they can do time series predictions with great efficiency.

\section{REFERENCES}

[1] Bo Han PhD Thesis, August 2011., Cognitive Routing for Wireless Ad Hoc Networks page 18 Department of Electronics, University of York

[2] Jyoti Prakash Singh, Paramartha Dutta, Arindrajit Pal. 2012 Delay prediction in Mobile Ad Hoc Network using Artificial Neural Network, Elsevier.

[3] Elizabeth M. Daly, Mads Haahr Pages 241-250 ScienceDirect 2009 The challenges of disconnected delay-tolerant MANETs, Elsevier.

[4] ByParkavi Murphy John I, Dr.P.Vivekanandan, 2012 A framework for Secure Routing in Mobile Ad hoc Networks. In Int. conf. On Advances in Engineering, Science And Management, IEEE.
[5] Qinghua Li, Sencun Zhu, Guohong Cao 2010 Routing in Socially Selfish Delay Tolerant Networks, IEEE INFOCOM

[6] Rakesh Kumar , Manoj Misra and Anil K. Sarje 2011 A Simplified Analytical Model for End-To-End Delay Analysis in MANET IJCA Special Issue on Mobile Adhoc Networks, IJCA.

[7] Nabhendra Bisnik, Alhussein A. Abouzeid 2007 Queuing network models for delay analysis of multihop wireless ad hoc networks, Elsevier.

[8] Zhihao Guo • Shaya Sheikh • Camelia Al-Najjar • Hyun Kim • Behnam Malakooti 2009 Mobile ad hoc network proactive routing with delay prediction using neural network- Springer.

[9] Heni kaaniche and Farouk kamoun 2010 Mobility Prediction in Wireless Ad Hoc Networks using Neural Networks pub in Journal Of Telecommunications.

[10] Mansour Sheikhan • Ehsan Hemmati 2011 Transient chaotic neural network-based disjoint multipath routing for mobile ad-hoc networks Springer.

[11] Hadi Noureddine, Qiang Ni, Geyong Min, Hamed AlRaweshidy 2010 A New Link Lifetime Prediction Method for Greedy and Contention-based Routing in Mobile Ad Hoc Networks. In 10th International Conference on Computer and Information Technology IEEE.

[12] Michele Garetto, and Emilio Leonardi, 2010 Restricted Mobility Improves Delay-Throughput Tradeoffs in Mobile Ad Hoc Networks, IEEE transactions on information theory, IEEE

[13] Jyoti Prakash Singh, Prabhat Kumar, Sunil Kumar Singh. 2012 Delay Prediction in Mobile Ad Hoc Network using Trapezoidal Fuzzy numbers. In $9^{\text {th }}$ Int conf. on computer science and software engineering IEEE.

[14] Ghadimi , A. Khonsari, A. Diyanat, M. Farmani , N. Yazdani 2011 An analytical model of delay in multi-hop wireless ad hoc networks pub in Science+Business Media Springer.

[15] Taesoo Jun, Nirmalya Roy, and Christine Julie 2010 Modelling Delivery Delay for Flooding in Mobile Ad Hoc Networks Communications (ICC),IEEE International Conference

[16] Nenad S. Kojić, Marija B. Zajeganović Ivančić, Irini S. Reljin and Branimir 2010 New algorithm for packet routing in mobile ad-hoc networks, journal of automatic control, university of Belgrade.

[17] M.Gholami, N.Cai, R.W.Brennan 2012 An artificial neural network approach to the problem of wireless sensors network localization Elsevier.

[18] Oscar F. Gonzalez, Michael Howarth, and George Pavlou 2007 Detection of Packet Forwarding Misbehavior in Mobile Ad-Hoc Networks Center for Communications Systems Research Springer.

[19] David Palma Helder Araujo, Marilia Curado 2012 Link quality estimation in wireless multi-hop networks using Kernel based methods, journal of computer networks Elsevier.

[20] A Chintawar, M Chatterjee, A Vidhate 2011 AOMDVAPLP: An Enhanced Algorithm with Accessibility and Link Breakage Prediction International Conference and Workshop on Emerging Trends in Technology (TCET), ACM.

[21] Soufiene Djahel, Farid Na"it-abdesselam, and Zonghua Zhang 2011 Mitigating Packet Dropping Problem in Mobile Ad Hoc Networks: Proposals and Challenges IEEE communications surveys, IEEE. 